\title{
OMORGUS (OMORGUS) SUBEROSUS (FABRICIUS) (COLEOPTERA: TROGIDAE) EM ESTERCO DE GALINHAS POEDEIRAS DE SÃO JOÃO DA BOA VISTA, SP, BRASIL
}

\author{
W.D.Z. Lopes ${ }^{1}$, F.H. da Costa ${ }^{2}$, W.C.Z. Lopes $^{3}$, J.C. de C. Balieiro ${ }^{4}$, V.E. Soares ${ }^{1}$, Â.P. do Prado ${ }^{5}$
}

${ }^{1}$ Universidade Estadual Paulista, Faculdade de Ciências Agrárias e Veterinárias, Via de Acesso Prof. Paulo Donatto Castellani, s/no, CEP 14884-900, Jaboticabal, SP, Brasil. E-mail: wdzlopes@fcav.unesp.br

\section{RESUMO}

\begin{abstract}
A família Trogidae (Coleoptera) é cosmopolita e suas espécies têm hábitos principalmente necrófagos. Estudos comOmorgus (Omorgus) suberosus (Fabricius, 1775), em fezes de granja aviária devem ser realizados, uma vez que poucas informações sobre esta espécie, neste tipo de ambiente, estão disponíveis na literatura. Foi realizado um levantamento deste trogídeo em granja de aves poedeiras situada em São João da Boa Vista, SP, Brasil, entre janeiro de 2001 a dezembro de 2002, utilizando-se, para captura, armadilhas de solo, funil de Berlese-Tullgren e flutuação em água. Dos 2.322 espécimes de $O$. suberosus coletados, armadilhas de solo somaram $99,17 \%$ do total, $0,51 \%$ pelo funil de Berlese-Tullgren e apenas 0,30\% (sete espécimes) foram capturados pela flutuação em água. Aumento significativo $(\mathrm{P}<0,05)$ na captura de $O$. suberosus foi registrado no verão de 2001 / 2002, além do que a armadilha de solo mostrou ser o método mais apropriado $(\mathrm{P}<0,05)$ para capturar esta espécie.
\end{abstract}

PALAVRAS-CHAVE: Granja avícola, Insecta, Omorgus suberosus, Trogidae.

\section{ABSTRACT}

OMORGUS (OMORGUS) SUBEROSUS (FABRICIUS) (COLEOPTERA:TROGIDAE) INPOULTRY MANURE IN SÃO JOÃO DA BOA VISTA, SP, BRAZIL. The family Trogidae (Coleoptera) is cosmopolitan and its species are mainly necrophagous. Studies on Omorgus (Omorgus) suberosus (Fabricius, 1775) on excrements in poultry houses are needed due to the scarcity of published information on the species in this kind of environment. A survey for this trogid was carried out in a poultry house in São João da Boa Vista, SP, Brazil, from January 2001 to December 2002 using soil and water flotation traps and Berlese-Tullgren funnel. A total of 2,322 specimens of O. suberosus were collected, being $99.17 \%$ by soil trap, $0.51 \%$ by Berlese-Tullgren funnel and only $0.30 \%$ (7 specimens) by water flotation trap. A significant increase in the capture $(\mathrm{P}<0.05)$ of $O$. suberosus was registered in the summer of 2001/2002, and the soil trap proved to be the most appropriate method $(\mathrm{P}<0.05)$ for capture of this species.

KEY WORDS: Poultry house, Insecta, Omorgus suberosus, Trogidae.

\section{INTRODUÇÃO}

A família Trogidae (Coleoptera) é de distribuição cosmopolita sendo constituída por cerca de 300 espécies de hábito principalmente necrófago ( $\mathrm{CHOLTZ}$, 1986). Em sua maioria ocorrem em regiões áridas dos continentes do HemisférioSul. Tanto os adultos como as larvas geralmente são encontrados associados ao último estágio da decomposição de restos animais (PAyne, 1965), alimentando-se também em guano de morcegos (Mammalia, Chiroptera) em cavernas, ovos degafanhotos (Insecta, Orthoptera), larvas de moscas (Insecta, Diptera) e ainda em itens não usuais, como carpetes velhos, chapéus-de-feltro, almofadas de pele e crina de cavalo (Equus caballus (Linnaeus, 1758) Mammalia, Perissodactyla, Equidae) (Scholtz, 1990). Os ovos dos trogídeos são colocados no solo, variando a profundidade no substrato alimentar; após a eclosão, as larvas posicionam-se verticalmente para alimentarem-se (VAURIE, 1962). Possuem três estádios larvais, com duração total de, aproximadamente, quatro semanas. O estágio de pupa, dura aproximada-

\footnotetext{
${ }^{2}$ Médico Veterinário, Autônomo, São João da Boa Vista, SP, Brasil, ${ }^{3}$ Universidade de São Paulo, Faculdade de Medicina, Ribeirão Preto, SP, Brasil.

${ }^{4}$ Universidade de São Paulo, Faculdade de Zootecnia e Engenharia Alimentar, Pirassununga, SP, Brasil, ${ }^{5}$ UNICAMP, Departamento de Parasitologia, Campinas, SP, Brasil.
} 
mente, duas semanas. Algumas espécies são fortemente atraídas pela luz e produzem sons (VANEMDEN, 1948).

Alguns autores acreditam que determinados trogídeos sejam endêmicos em ninho de aves predadoras como corujas (Aves, Strigiformes), enquanto que outras espécies vêm sendo encontradas em toca de animais carnívoros (Mammalia, Carnivora) (Gomes, 2005).

Esta família é constituída por 3 gêneros: Trox Fabricius, 1775; Omorgus Erichson, 1847 com dois subgêneros, Omorgus Erichson, 1847 e Haroldomorgus Scholtz, 1986; e Polynoncus Burmeister, 1876. O gênero Trox é amplamente distribuído principalmente nas regiões Holártica e Afrotropical, já o gênero Omorgus ocorre normalmente em regiões áridas, principalmente nos continentes ao Sul e, por fim, o gênero Polynoncus que possui distribuição restrita à América do Sul.

Poucos trabalhos relacionados ao O. suberosus em granja de aves poedeiras foram publicados. Somente GIANIZELLA; PRADO (1999) relataram esta espécie neste tipo de excremento, o que, sem dúvida, dificulta um melhor entendimento do que realmente ocorre com este trogídeo neste ecossistema artificial.

Deste modo, o presente trabalho teve como objetivos estudar a sazonalidade doO.suberosus dentro das estações do ano e evidenciar a metodologia mais apropriada para captura deste artrópode no ecossistema artificial de esterco de aves poedeiras.

\section{MATERIAL E MÉTODOS}

Descrição da Granja: as coletas foram realizadas na granja Crisdan, situada a $7 \mathrm{~km}$ do Município de São João da Boa Vista, Estado de São Paulo (22 $01^{\prime}$ S, $46^{\circ} 48^{\prime} \mathrm{W}$, altitude de $763 \mathrm{~m}$ ).

A granja, considerada de pequeno porte, possuía 2 conjuntos de 4 galpões (abertos) cada, com capacidade para alojar 25.000 galinhas Gallus gallus domesticus (Linnaeus, 1758) - Aves, Galliformes, Phasianidae) das linhagens "Hy Line" e "Hy-line Brown", em fase de postura.

O galpão de coleta possuía 3 conjuntos de gaiolas (ou 3 corredores onde se acumulam as fezes) separados por dois corredores de concreto de 0,5 m de largura. As fileiras das gaiolas estavam distribuídas em degrau (tipo "narrow house"). As gaiolas variavam de tamanho e abrigavam de duas a quatro galinhas. Sob as gaiolas, o piso era de terra onde as fezes se acumulavam.

Ao redor da granja havia culturas agrícolas e, durante o período analisado, a predominância foi de café (Coffea sp. - Rubiaceae) e milho(ZeamaysL. - Poaceae). Durante a coleta, nenhum inseticida foi utilizado sobre o esterco, o qual foi retirado em 24/ 4 e 20/9 de 2001 e em 15/3 e 2/11 de 2002. Devido às altas precipitações pluviométricasconstatadas noverão, ouso deóxidode cálcio e de serragem foi relativamente comum.

Foram realizadas 75 coletas entre 17/1/2001 a $26 / 12 / 2002$, com intervalos de sete dias durante os primeiros 12 meses, passando a quinzenais nos demais meses, totalizando 1.125 armadilhas e $600 \mathrm{~kg}$ de esterco. Para amostragem dos espécimes adultos foram utilizados três métodos distintos: armadilhade-solo (método 1), o funil de Berlese-Tullgren (método 2) e a flutuação em água (método 3).

Método1 - Armadilha-de-solo: estas armadilhas foram utilizadas visandoa obtenção de trogídeos adultos (Walker, 1985; Summerlin, 1989). Consistiram de frascos plásticos com 9,0 cm de altura e $8,0 \mathrm{~cm}$ de diâmetro contendo $200 \mathrm{~mL}$ de líquido fixador e conservante $(80 \%$ de água; $5 \%$ de glicerina; $5 \%$ de álcool a $70 \%$; $5 \%$ de formol e $5 \%$ de detergente) para posterior identificação e quantificação em microscópio esterioscópio. As armadilhas foram retiradas e substituídas por outras com novo fixador em cada coleta, sendo elas numeradas de 1 a 15 , as quais foram escolhidas aleatoriamente e, posteriormente, enterradas sob as gaiolas, ao longo de 3 corredores (5/ armadilhas/corredor) A, BeC, próximo ao esterco acumulado.

A distribuição das armadilhas foi feita por "amostragem sistemática", que consistiu em delimitar áreas em quadrados contíguos $\left(1 \mathrm{~m}^{2}\right)$, onde elas foram distribuídas (KREBS, 1989).

Método 2 - Extração direta do esterco: o esterco acumulado sob as gaiolas apresentava, visualmente, cinco tipos distintos de consistência: líquido, pastoso, pastoso-firme, firme e seco (BRUNO et al., 1993), sendo, que no galpão, havia esterco com uma ou mais consistências diferentes. As amostras foram retiradas de forma a obter esterco de todas as consistências.

$\mathrm{Na}$ coleta das amostras de esterco foi utilizada uma pá, ao invés da introdução de um cilindro de metal, como recomendado porPECK; ANDERSON (1969). Para obtenção deste material, foram extraídas cerca de 10 (400 g/ amostra) amostras aleatórias num total de, aproximadamente, $4 \mathrm{~kg}$ dos diferentes tipos de esterco por coleta.

Em seguida, o material coletado foi transferido para funil de Berlese-Tullgren confeccionado com um tubo de papel cartolina sobre um vidro, com líquido fixador e conservador (álcool a 70\%). Sobre o funil foi utilizada uma lata tipo embalagem de achocolatado sem fundo que foi substituída por uma tela de malha de $1 \mathrm{~cm}$. O esterco (cerca de $670 \mathrm{~g} /$ funil) era colocado dentro da lata e ficava exposto a lâmpada de $25 \mathrm{~W}$, durante 5 dias.

Método 3 - Flutuação em água:as amostras foram extraídas do esterco utilizando-se o método de Roberts recomendado por MoORe (1954), com base no princípio de flutuação. 
Da mesma forma que o método anterior, cerca de 10 amostras (400 g/amostra) foram extraídas aleatoriamentedogalpão para obtenção domaterial, totalizando, aproximadamente, $4 \mathrm{~kg}$ de esterco por coleta.

Em 8 baldes de 10 L decapacidade, contendoágua quase até a borda, foram colocadas porções de cerca de $500 \mathrm{~g}$ de esterco/balde. Após agitação feita com o auxílio de um bastão de madeira e posterior decantação do esterco, foram coletados os artrópodes que flutuavam, com o auxílio de uma peneira de malha fina. Os espécimes obtidos foram secados em papel toalha, para posterior identificação.

Todo material coletado foi levado para o laboratório onde foi triado, contado, identificado, fixado e conservado em álcool a 70\%, sendo que, para a identificação do Omorgus (Omorgus) suberosus, foi utilizado o trabalho de Scholtz (1990). O material testemunho encontra-se na Coleção Entomológica da Faculdade de Medicina Veterinária do Centro Universitário da Fundação de Ensino "Octávio Bastos" (UNIFEOB), São João da Boa Vista, SP.

Os dados desazonalidadereferentes aos 3 métodos de coleta ao longo dos dois anos de coleta foram previamente transformados em $\log (x+1)$ e, posteriormente, efetuou-sea análise de variância por meio deum delineamento em parcela subdividida no tempo("Split Plot in Time"), sendo estabelecidos como tratamento principal os métodos de coleta e, como tratamento secundário, as estações dos anos para obtenção das significâncias do teste F. As médias $(=S[\log (x+1)] /$ n) dos tratamentos foram obtidas pelo procedimento Lsmeans utilizando o teste Tukey-Kramer $(\mathrm{P}<0,05)$.

As análises das correlações momento-produto de Pearson foram utilizadas para avaliação de quão associados foram os números deO. suberosuscapturados e temperatura (ambiente), umidade relativa do ar eosíndices pluviométricos. Os dados pluviométricos, de temperatura e umidade relativa do ar foram fornecidos pelo Banco de Dados Hidrometerológicos da Cooperativa Agrícola Nacional Sudeste Centro Oeste, situada em São João da Boa Vista, SP. O teste para verificação de correlações realizada pelo programa anteriormente citado é um Teste $t$ de Student.

Para tanto, foram utilizados os procedimentos GLM do programa Statistical Analysis System, versão 6.12.

\section{RESULTADOS E DISCUSSÃO}

Capturaram-se 2.322 espécimes de trogídeos adultos pertencentes à espécie $O$. (Omorgus) suberosus associados ao esterco de galinhas poedeiras. Utilizando-se armadilhas de solo, foram coletados 2.303 exemplares $(99,17 \%$ do total); nas coletas realizadas por meio do funil de Berlese-Tullgren, foram encontrados 12 trogídeos $(0,51 \%)$ e, pela flutuação em água, apenas sete $(0,30 \%)$. Esta espécie possui de 11,5a 15,0 mm de comprimento e 6,8 a $8 \mathrm{~mm}$ de largura, sendo amplamente distribuída no Novo Mundo, desde o Canadá até o sul da Argentina. BAKER (1968) relatou que O. suberosus preda ovos de gafanhotos na América do Sul.

Poucas informações sobre os hábitos alimentares do O. suberosus na América doSul existem na literatura, porém, observações de pesquisadores indicam que eles ocorrem em situações típicas da família Trogidae, tais como carcaças, pêlos e em acúmulo de fezes de granja aviária (PAYNE, 1965).

Tabela 1 - Resultado das comparações múltiplas (médias ajustadas - Tukey-Kramer) do desdobramento da interação estações do ano (2001 e 2002) e métodos de captura, em granja aviária de São João da Boa Vista, SP.

\begin{tabular}{|c|c|c|c|c|}
\hline \multirow[t]{2}{*}{ Armadilhas } & \multicolumn{4}{|c|}{ Estações do ano/médias $=\mathrm{S}[\log (\mathrm{x}+1) / \mathrm{n}]$ de Omorgus (Omorgus) suberosus } \\
\hline & Verão & Outono & Inverno & Primavera \\
\hline \multirow{3}{*}{ Armadilhas de solo } & $\mathrm{A}$ & A & A & A \\
\hline & 1,6869 & 1,3885 & 1,3208 & 1,0992 \\
\hline & a & $\mathrm{b}$ & bc & $\mathrm{c}$ \\
\hline \multirow{3}{*}{ Funil de Berlese-Tullgren } & B & B & $\mathrm{B}$ & B \\
\hline & 0,0215 & 0,0628 & 0,0741 & 0,0000 \\
\hline & $\mathrm{a}$ & $a$ & $\mathrm{a}$ & $\mathrm{a}$ \\
\hline \multirow{3}{*}{ Flutuação em água } & B & $\mathrm{B}$ & B & B \\
\hline & 0,0645 & 0,0301 & 0,0143 & 0,0151 \\
\hline & $\mathrm{a}$ & $\mathrm{a}$ & $\mathrm{a}$ & $\mathrm{a}$ \\
\hline
\end{tabular}

*Médias seguidas por pelo menos uma letra em comum, minúscula na linha e maiúscula na coluna não diferem entre si pelo teste Tukey $(\mathrm{P}>0,05)$.

* = Significativo $(\mathrm{P}<0,05)$

** $=$ Significativo $(\mathrm{P}<0,01)$ 
Tabela 2 - Total de Omorgus (Omorgus) suberosus capturados nas diferentes estações do ano, segundo o método de amostragem, em granjas de aves poedeiras de São João da Boa Vista, SP.

\begin{tabular}{lccrr}
\hline Estações dos anos (2001e 2002) & Armadilhas de solo & Funil de Berlese-Tullgren & $\begin{array}{c}\text { Flutuação } \\
\text { em água }\end{array}$ & Total \\
\hline Primavera & 371,00 & 0,00 & 1,00 & 372,00 \\
Verão & 833,00 & 1,00 & 3,00 & 837,00 \\
Outono & 591,00 & 5,00 & 2,00 & 598,00 \\
Inverno & 500,00 & 12,00 & 3,00 & 515,00 \\
\hline Total & $2.295,00$ & 18,00 & 9,00 & $2.322,00$ \\
\hline
\end{tabular}

Tabela 3 - Valores médios das covariáveis temperatura $\left({ }^{\circ} \mathrm{C}\right)$, umidade realtiva $(\%)$ e pluviometria $\left(\mathrm{mm}^{3}\right)$ ao longo das estações do ano de 2001 e 2002.

\begin{tabular}{lccc}
\hline Estações dos anos (2001 e 2002) & $\begin{array}{l}\text { Temperatura }\left({ }^{\circ} \mathrm{C}\right) \\
\text { média/estação }\end{array}$ & $\begin{array}{l}\text { Umidade relativa (\%) } \\
\text { média/estação }\end{array}$ & $\begin{array}{l}\text { Pluviosidade }\left(\mathrm{mm}^{3}\right) \\
\text { total/estação }\end{array}$ \\
\hline Primavera & $27,20^{\mathrm{ns}}$ & $70,45^{\mathrm{ns}}$ & $5.183^{\mathrm{ns}}$ \\
Verão & $27,85^{\mathrm{ns}}$ & $70,64^{\mathrm{ns}}$ & $2.649^{\mathrm{ns}}$ \\
Outono & $25,60^{\mathrm{ns}}$ & $67,85^{\mathrm{ns}}$ & $1.459^{\mathrm{ns}}$ \\
Inverno & $25,05^{\mathrm{ns}}$ & $57,42^{\mathrm{ns}}$ & $1.201^{\mathrm{ns}}$ \\
\hline
\end{tabular}

Coeficiente de correlação das covariáveis Temperatura (TEMP), Umidade relativa (UR) e Pluviometria (PP) para O. suberosus capturados em armadilhas de solo, flutuação em ágrua e funil de Berlese-Tullgren.

\begin{tabular}{|c|c|c|c|}
\hline & $\operatorname{TEMP}\left({ }^{\circ} \mathrm{C}\right)$ & UR (\%) & $\mathrm{PP}\left(\mathrm{mm}^{3}\right)$ \\
\hline Omorgus (Omorgus) suberosus & $0,07448^{\text {ns }}$ & $-0,16858^{\text {ns }}$ & $0,15009^{\text {ns }}$ \\
\hline
\end{tabular}

$\overline{\mathrm{ns}}=$ Não Significativo $(\mathrm{P}>0,05)$

Tabela 4 - Resultado das comparações múltiplas (médias ajustadas - Tukey-Kramer ) do desdobramento da interação corredor e número da armadilha de captura, em granja aviária de São João da Boa Vista, SP.

\begin{tabular}{lccc}
\hline$N^{o}$ da armadilha de solo & Corredor A & Corredor B & Corredor C \\
\hline Armadilha 1 & $21,271^{\mathrm{A}}$ & $21,1390^{\mathrm{A}}$ & $18,2610^{\mathrm{A}}$ \\
Armadilha 2 & $21,303^{\mathrm{A}}$ & $23,0320^{\mathrm{A}}$ & $20,5690^{\mathrm{A}}$ \\
Armadilha 3 & $24,048^{\mathrm{A}}$ & $25,9440^{\mathrm{A}}$ & $18,9760^{\mathrm{A}}$ \\
Armadilha 4 & $18,261^{\mathrm{A}}$ & $20,7190^{\mathrm{A}}$ & $23,9790^{\mathrm{A}}$ \\
Armadilha 5 & $20,864^{\mathrm{A}}$ & $18,2610^{\mathrm{A}}$ & $22,7180^{\mathrm{A}}$ \\
\hline Médias $=\mathrm{S}[\log (\mathrm{x}+1) / \mathrm{n}]$ & $21,149^{\mathrm{a}}$ & $21,819^{\mathrm{a}}$ & $20,901^{\mathrm{a}}$ \\
\hline
\end{tabular}

*Médias seguidas por pelo menos uma letra em comum, minúscula na linha e maiúscula na coluna não diferem entre si pelo Teste Tukey $(\mathrm{P}>0,05)$.

O único relato de O. suberosus encontrado associado ao esterco de aves poedeiras foi realizado por GiANIZELLA; PRADO (1999) em uma granja comercial de Monte Mor, Estado de São Paulo.

Em esterco de aves poedeiras, a armadilha de solo parece ser o método mais adequado $(\mathrm{P}<0,05)$ para capturar O. suberosus em relação ao funil de BerleseTullgren e a flutuação em água (Tabela 1). Acreditase que a baixa freqüência de $O$. suberosus, capturados no funil de Berlese-Tullgren e pela flutuação emágua, seja decorrente do fato que este coleóptero possui uma maior biomassa (FLECHTMAN et al., 1995) em relação a outros artrópodes (comumente capturados por estes métodos), dificultando desta maneira a sua captura pelos métodos supracitados.

O acme populacional de O. suberosus capturados pelos três métodos de coleta foi em fevereiro de 2001 (363), quando a temperatura média neste mês foi de $29^{\circ} \mathrm{C}$ (Fig. 1). A menor ocorrência pode ser constatada em outubro de 2002 com apenas quatro trogídeos registrados. Larvas da espécie não foram encontradas durante os 24 meses de captura. O mesmo pode ser observado por GiAnIZELLA; PRADO (1999). Estes autores estudaram a sazonalidade desta espécie de Trogidae 
capturados por armadilha de solo, notificando grandes picos populacionais entre os meses de setembro e dezembro, nos 2 anos em que este trogídeo foi coletado. No presente estudo, convém salientar que foi constatado um aumento significativo $(\mathrm{P}<0,05)$ no número de espécimes deO.suberosus, capturados pormeiodearmadilhas desolono verão de2001/2002, eque, noinverno, ocorreu uma queda abrupta $(\mathrm{P}<0,05)$ (Tabelas 1 e 2$)$.

As correlações entre os totais de exemplares de $O$. suberosus encontrados e dados abióticos (temperatura umidade relativa do ar e precipitação pluvial) foram de baixa magnitude, indicando baixas associações com estas covariáveis. A temperatura durante o período de coleta apresentou média mínima de $25,05^{\circ} \mathrm{C}$ (inverno de 2001 e 2002) e máxima de $27,85^{\circ} \mathrm{C}$ no verão de ambos os anos amostrados, umidade relativa do ar apresentou média mínima de $57,42 \%$ no inverno e máxima de $70,64 \%$ no verão de 2001 e 2002, para pluviosidade foi notificada somatória mínima de $1.201 \mathrm{~mm}^{3}$ nosmeses de inverno dos anos de2001 e2002emáxima de $5.183 \mathrm{~mm}^{3}$ na primavera dos anos levantados (Tabela 3).

É importante ressaltar que não houve diferenças significativas $(\mathrm{P}>0,05)$ no total de espécimes de $O$. suberosus amostrados nos diferentes corredores (A, B eC) em que as armadilhas de solo estiveram dispostas (5/armadilhas/corredor) aleatoriamente (Tabela 4). Entretanto, sua presença ao longo do galpão de coleta sempre esteve associada a locais onde havia grande quantidade de ração, ovos quebrados e penas que eram freqüentemente derrubadas pelas aves. Além disso, sua coloração produziu grande efeito mimético, fazendo-o, muitas vezes, passar despercebido no esterco, mesmo estando algumas vezes em grande densidade populacional.

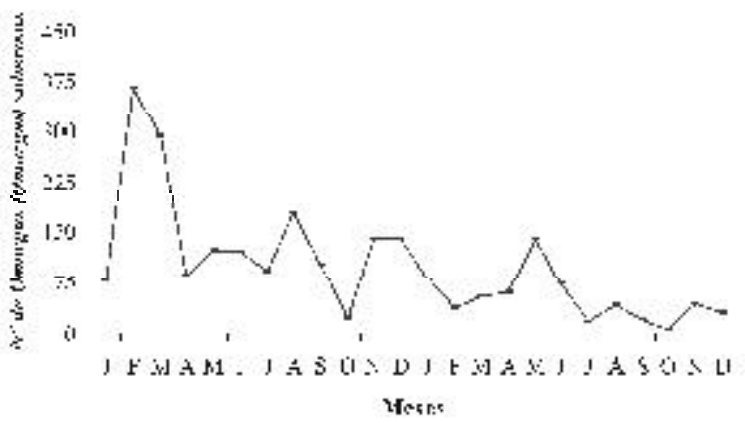

Fig. 1 - Total de espécimes deOmorgus (Omorgus)suberosus (Fabricius, 1775) (Coleoptera, Trogidae) capturados por armadilhas de solo, funil de Berlese-Tullgren e flutuação em água, de janeiro de 2001 a dezembro de 2002, em granja aviária de São João da Boa Vista, SP, Brasil.

\section{CONCLUSÕES}

Osresultadosencontrados possibilitamconcluirque:
- além do método de captura, as estações do ano e a precipitação pluvial, outros fatores como os componentes genéticos, interação social e capacidade de dispersão desta espécie de coleóptero podem estar interagindo na sazonalidade de O. suberosus;

- armadilhas de solo mostraram ser a metodologia mais apropriada para capturar $O$. suberosus neste ecossistema artificial de aves poedeiras.

\section{AGRADECIMENTOS}

Ao Dr. Gilson Pereira de Oliveira, Dra. Marisa Rossi Monteiro pela colaboração técnica, aos professores Maria Cândida de Oliveira Costa e Afonso Celso Navarro pelas sugestões e comentários. Este trabalho foi financiado pela UNIFEOB.

\section{REFERÊNCIAS}

BAKER, C.W. Larval taxonomy of the trogine with notes on biologies and life histories. Bulletin of the United States National Museum, v.279, p.1-79, 1968.

Bruno, T.V.; Guimarães, J.H.; Santos, A.M.M.; Tucci, E.C. Moscas sinantrópicas (Diptera) e seus predadores que se criam em esterco de aves poedeiras confinadas, no Estado de São Paulo, Brasil. Revista Brasileira de Entomologia, v.37, n.2, p.577-590, 1993.

EMDEN, F.I. van. Trox larva feeding on locust eggs in Somalia. Proceedings of the Royal Entomological Society of London, v.17, n.3, p.145-148, 1948.

Flechtman, C.A.H.; RodRIGUes, S.R.; Couto, H.T.Z. Controle biológico da mosca-dos-chifres (Haematobia irritans) em Selvíria, Mato Grosso do Sul. Levantamento de espécies fimícolas associadas à mosca. Revista Brasileira de Entomologia, v.39, n.1, p.249-258, 1995.

Gianizella, S.L.; Prado, A.P. Ocorrência e sazonalidade de Omorgus (Omorgus) suberosus (Fabr.) (Trogidae: Coleoptera) em esterco de aves poedeiras em Monte Mor, SP. Anais da Sociedade Entomológica do Brasil, v.28, n.3, p.749-751, 1999.

GOMEs, R.S. Atractividad de diferentes cebos sobre trogídos (Coleoptera) en el bosque autóctono "El Espinal", Río Cuarto (Córdoba, Argentina). Revista de la Sociedad Entomológica Argentina, v.64, n.2, p.103-105, 2005.

KREBS, C.J. Ecological methods. New York: Harper \& Row, 1989. 700p.

Moore, I. An efficient method of collecting dung beetles. Pan-Pacific Entomologist, v.30, n.4, p.208, 1954.

PAYNE, J.A.A summer carrion study of the baby pig Sus scrofta Linnaeus. Ecology, v.46, n.5, p.592-602, 1965.

Peck, R.F.; Anderson, J.R. Arthropod predators of immature Diptera developing in poultry groupings in northern California. Journal of Medical Entomology, v.6, n.1, p.163167, 1969.

Scholtz, C.H. Phylogeny and systematics of the Trogidae (Coleoptera: Scarabaeoidea). Systematic Entomology, v.11, p.355-363, 1986. 
Scholtz, C.H. Revision of the Trogidae of South America. Journal of Natural History, v.24, p.1391-1456, 1990.

SummerLIN, J.W. Tetchiness for collecting, rearing and handling histerid beetles. Southwestern Entomologist, v.14, p.415-425, 1989.

VAURIE, P. A revision of the genus Trox in South America (Coleoptera: Scarabaeidae). Bulletin of the American Museum of Natural History, v.124, n.4, p.101-168, 1962.
Walker, R.L. A pitfal trap study on the Carabidae and Staphylinidae (Coleoptera) in Country Durham. Entomologist's Monthly Magazine, v.121, n.1, p.1-18, 1985.

Recebido em 27/9/06

Aceito em 23/8/07 